\title{
IN SIMPLY CONNECTED COTANGENT BUNDLES, EXACT LAGRANGIAN COBORDISMS ARE H-COBORDISMS
}

\author{
HIRO LEE TANAKA
}

\begin{abstract}
We show that if $\mathrm{Q}$ is simply connected, then every exact Lagrangian cobordism between compact, exact Lagrangians in the cotangent bundle of $\mathrm{Q}$ is an h-cobordism. The result follows as a corollary of the AbouzaidKragh theorem.
\end{abstract}

\section{INTRODUCTION}

We prove:

Theorem 1. Let $Q$ be simply connected and smooth. Any compact exact Lagrangian cobordism between compact, exact Lagrangians in $T^{*} Q$ is an $h$-cobordism.

By the $h$-cobordism theorem [Sma62, Mil65], we have:

Corollary 2. If $Q$ is smooth, simply connected, and has $\operatorname{dim} Q \geq 5$, any two compact, exact Lagrangians related by an exact compact cobordism are diffeomorphic.

This result is motivated by two topics of interest. The first is the Nearby Lagrangian Conjecture of Arnol'd [Arn86], which conjectures that if $Q$ is a smooth, compact manifold (not necessarily simply connected), then any compact, exact Lagrangian in $T^{*} Q$ is Hamiltonian-isotopic to the zero section. Theorem 1 shows that the classification of Lagrangian cobordism classes in $T^{*} Q$ provides another strategy for attacking the conjecture when $\operatorname{dim} Q \geq 5$ and $\pi_{1} Q=*$. Namely, if there is only one exact, compact cobordism class, then every compact exact Lagrangian is cobordant to the zero section by a cylinder. An unknotting theorem for cylindrical cobordisms in $T^{*}(Q \times \mathbb{R})$ would exhibit the cobordism as a Hamiltonian isotopy.

The second is recent work on categories of Lagrangian cobordisms, as developed in [NT11] and [BC13a, BC13b]. For a fixed symplectic manifold $M$ satisfying certain monotonicity or convexity conditions, one can define a category whose objects are Lagrangians (equipped with standard Floer-theoretic decorations) and whose morphisms are Lagrangian cobordisms between them (equipped with compatible decorations). In the setting of [NT11], all objects and cobordisms are exact. The theorem shows one motivation for considering non-compact cobordisms in this category. (Otherwise, every morphism is homotopically uninteresting.)

Finally, there is another way in which Theorem 1 touches both topics. Recall:

Theorem 3 (Fukaya-Nadler-Seidel-Smith [Nad09, FSS08]). Let $Q$ be smooth, compact, and simply connected. Any compact exact Lagrangian inside $T^{*} Q$ is equivalent to $Q$ in the Fukaya category of $T^{*} Q$, with $\mathbb{Z} / 2 \mathbb{Z}$ coefficients. If both $Q$ and the Lagrangian are Spin, then the same is true over arbitrary coefficients.

Date: June 22, 2018.

Key words and phrases. Cobordisms, Fukaya Categories, Mirror Symmetry. 
It can be shown that compact, exact Lagrangians related by a compact, exact cobordism are equivalent in the Fukaya category [Tan13, Tan]. ${ }^{1}$ Hence the equivalence class of an object in Fukaya $\left(T^{*} Q\right)$ is an invariant of an exact Lagrangian's cobordism class. If this is a complete invariant (in the way Stiefel-Whitney numbers classify unoriented cobordism classes) the above theorem of Fukaya-NadlerSeidel-Smith, together with Theorem 1, would show that any two exact, compact Lagrangians are $h$-cobordant (to the zero section).

Remark 4. Finally, we are told that Lara-Simone Suarez has a result for nonsimply-connected cobordisms [Sua] - specifically, that exact, spin, Lagrangian cobordisms for which the collar inclusions induce isomorphisms on $\pi_{1}$ are diffeomorphic to cylinders. Instead of relying on Abouzaid-Kragh's theorem, she utilizes a previous result of Biran and Cornea [BC13a]. This also allows her to consider symplectic manifolds that need not be cotangent bundles.

1.1. Acknowledgements. We are grateful to Tim Perutz for helpful feedback on this paper. The author was supported by a Presidential Fellowship from Northwestern University's Office of the President, an NSF Graduate Research Fellowship, and a Mathematical Sciences Research Institute Postdoctoral Fellowship.

\section{ReCOlLECtions}

Recall that $T^{*} Q$ has a 1 -form $\theta_{Q}=\sum_{i} p_{i} d q_{i}$ whose derivative is symplectic.

Definition 5. We say a Lagrangian submanifold $L \subset T^{*} Q$ is exact if it is equipped with a smooth function $f_{L}: L \rightarrow \mathbb{R}$ for which $d f_{L}=\left.\theta\right|_{L}$. We call $f_{L}$ a primitive for $L$.

Definition 6. A Lagrangian submanifold $W \subset T^{*} Q \times T^{*}(0,1)$ is said to be a Lagrangian cobordism from $L_{0}$ to $L_{1}$ if

$$
\left.W\right|_{(0, \epsilon)}=L_{0} \times(0, \epsilon) \quad \text { and }\left.\quad W\right|_{(1-\epsilon, 1)}=L_{1} \times(1-\epsilon, 1) \subset M \times T^{*}(0,1) .
$$

Fixing primitives $f_{L_{i}}$, we say $W$ is exact if one can choose a function $f_{W}: W \rightarrow \mathbb{R}$ so that

$$
\left.f_{W}\right|_{L_{0} \times(0, \epsilon)}=\left.f_{L_{0}} \quad f_{W}\right|_{L_{1} \times(1-\epsilon, 1)}=f_{L_{1}}, \quad d f_{W}=\left.\left(\theta_{Q}+\theta_{\mathbb{R}}\right)\right|_{W} .
$$

In particular, the value of $f$ is independent of $t$ when $t \in(0, \epsilon) \bigcup(1-\epsilon, 1)$.

Finally, recall the following:

Theorem 7 (Abouzaid-Kragh [Abo12, Kra13]). If $L \subset T^{*} Q$ is a compact, exact Lagrangian, then the projection map $L \rightarrow Q$ is a homotopy equivalence.

\section{Proof}

Given a (not necessarily Lagrangian) cobordism $Z$, let $Z^{\text {op }}$ be the same cobordism with ingoing and outgoing boundaries interchanged. We call the composite $Z \circ Z^{\mathrm{op}}$ a double of $Z$. (The other double is the composition $Z^{\mathrm{op}} \circ Z$.)

Lemma 8. Let $Q$ be simply connected. Then any compact, exact cobordism between two compact exact Lagrangians in $T^{*} Q$ has a double which is an $h$-cobordism.

\footnotetext{
${ }^{1}$ Over arbitrary coefficients, one needs each Lagrangian to have a Spin structure, and for the cobordism to respect this structure. In characteristic 2, no such structure is needed.
} 
Lemma 9. Let $Y_{0}, Y_{1}$ be simply connected manifolds. If $Z$ is any smooth cobordism between them such that a double is an $h$-cobordism, then $Z$ is an $h$-cobordism itself.

Proof of Theorem. By Abouzaid-Kragh, any two compact exact Lagrangians in $T^{*} Q$ are homotopy equivalent to $Q$, so are simply connected. By the lemmas, any compact exact cobordism between them is an $h$-cobordism.

Proof of Lemma 8. Let $Y_{01} \subset T^{*} Q \times T^{*}(0,1)$ be a cobordism from $Y_{0}$ to $Y_{1}$, with each $Y_{i} \subset T^{*} Q$ compact and exact. Note that the diffeomorphism $\mathbb{R} \rightarrow \mathbb{R}$ given by $t \mapsto-t$ gives rise to another exact cobordism $Y_{01}^{\mathrm{op}} \subset M \times T^{*}(0,1)$, from $Y_{1}$ to $Y_{0}$. We prove that the composite cobordism

$$
N=Y_{01} \circ\left(Y_{01}\right)^{\text {op }}
$$

is an $h$-cobordism.

Note that since $N$ is a cobordism collared by $Y_{1}$ on both ends, we can glue the $Y_{1}$ on both ends via the identity to obtain a compact, exact Lagrangian in $T^{*}\left(Q \times S^{1}\right)$. (We are guaranteed exactness since, by definition of cobordism, the primitive function $f$ realizing $d f=\theta_{Y_{1}}$ must agree along the collars). We call this Lagrangian $\bar{N}$.

By Abouzaid-Kragh, the projection map $\bar{N} \rightarrow Q \times S^{1}$ is a homotopy equivalence. Consider the diagram

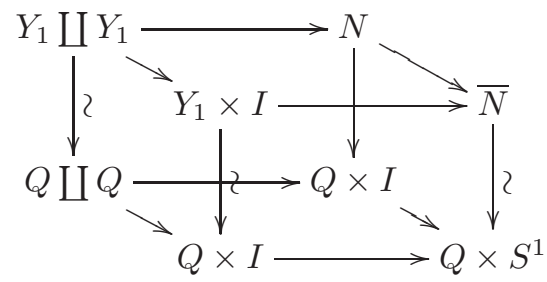

where the top and bottom faces are pushout squares. (The all vertical arrows are projection maps to the zero section, and the indicated arrows are equivalences by Abouzaid-Kragh.) By excision and the Five Lemma, the remaining vertical arrow induces an isomorphism in homology $H_{*}(N) \rightarrow H_{*}(Q \times I)$.

Moreover, $N$ has trivial fundamental group: the groupoid version of van Kampen's theorem shows there must be a pushout diagram of groupoids

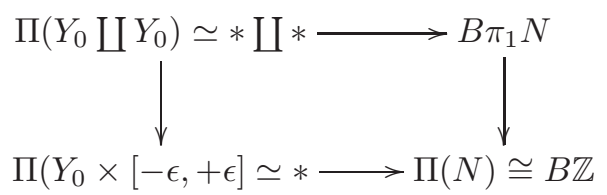

whence it follows that $\mathbb{Z} \cong \mathbb{Z} * \pi_{1} N$, hence $\pi_{1} N \cong 0$. By the Whitehead and Hurewicz theorems, the projection map thus realizes a homotopy equivalence $N \simeq$ $Q \times I$. Moreover, the inclusion of $Y_{1}$ on either collar of $N$ fits into a commutative diagram of topological spaces

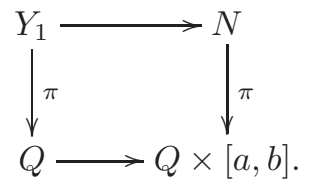


The lefthand vertical arrow is a homotopy equivalence by the Abouzaid-Kragh theorem, as is the righthand vertical arrow from our previous discussion. The bottom horizontal arrow is obviously a homotopy equivalence, so the top horizontal arrow must be as well. This shows that $N$ is in fact an $h$-cobordism from $Y_{1}$ to itself.

Proof of Lemma 9. Note we have the inclusion $Y_{0} \hookrightarrow Y_{01} \circ\left(Y_{01}\right)^{\text {op }}$. We make use of the pushout diagram

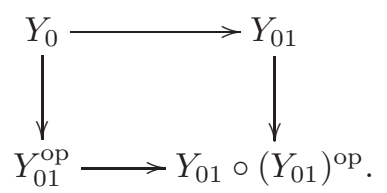

Knowing that $Y_{0}$ is simply connected and that $Y_{1} \simeq Y_{01}^{\mathrm{op}} \circ Y_{01}$ is simply connected, the Van Kampen theorem shows that $Y_{01}$ is also simply connected. Finally, the Mayer-Vietoris sequence splits at each level by including $Y_{1} \rightarrow Y_{01}$. Thus the Whitehead and Hurewicz theorems show that $Y_{01}$ is an $h$-cobordism.

\section{REFERENCES}

[Abo12] Mohammed Abouzaid, Nearby Lagrangians with vanishing Maslov class are homotopy equivalent, Invent. Math. 189 (2012), no. 2, 251-313. MR 2947545

[Arn86] V. I. Arnol'd, The first steps of symplectic topology, Uspekhi Mat. Nauk 41 (1986), no. 6(252), 3-18, 229. MR 890489 (89d:58034)

[BC13a] Paul Biran and Octav Cornea, Lagrangian cobordism. I, J. Amer. Math. Soc. 26 (2013), no. 2, 295-340. MR 3011416

[BC13b] L Lagrangian cobordism. II, arXiv:1304.6032, 2013.

[FSS08] Kenji Fukaya, Paul Seidel, and Ivan Smith, Exact Lagrangian submanifolds in simplyconnected cotangent bundles, Invent. Math. 172 (2008), no. 1, 1-27. MR 2385665 (2009a:53142)

[Kra13] Thomas Kragh, Parametrized ring-spectra and the nearby Lagrangian conjecture, Geom. Topol. 17 (2013), no. 2, 639-731, With an appendix by Mohammed Abouzaid. MR 3070514

[Mil65] John Milnor, Lectures on the h-cobordism theorem, Notes by L. Siebenmann and J. Sondow, Princeton University Press, Princeton, N.J., 1965. MR 0190942 (32 \#8352)

[Nad09] David Nadler, Microlocal branes are constructible sheaves, Selecta Math. (N.S.) 15 (2009), no. 4, 563-619. MR 2565051 (2010m:53131)

[NT11] David Nadler and Hiro Lee Tanaka, A stable $\infty$-category of Lagrangian cobordisms, arXiv:1109.4835, 2011

[Sma62] S. Smale, On the structure of manifolds, Amer. J. Math. 84 (1962), 387-399. MR 0153022 (27 \#2991)

[Sua] Lara-Simone Suarez, Exact Lagrangian cobordism and pseudo-isotopy, Ph.D. Thesis (in preparation), Universite de Montreal.

[Tan] Hiro Lee Tanaka, A functor showing cobordant Lagrangians are equivalent in Fukaya(M), in preparation.

[Tan13] — A functor from Lagrangian cobordisms to the Fukaya category, Ph.D. Thesis, Northwestern University, 2013.

One Oxford Street, Cambridge, MA, 02138

E-mail address: hirolee@math.harvard.edu 